\title{
Probing the constitutive behaviour of an Al-Sn-Si alloy by tensile testing and instrumented indentation
}

\author{
R. Schouwenaars, H. A. Durán, A. Bravo, V. H. Jacobo \& A. Ortiz \\ Departamento de Materiales y Manufactura, Facultad de Ingeniería, \\ Universidad Nacional Autónoma de México, Mexico
}

\begin{abstract}
Al-Sn-Si alloys used in tribological applications present a composite microstructure consisting of the Al-matrix, Sn-grains and dispersed $\mathrm{Si}$ and intermetallic particles. Upon plastic deformation, compatibility strains at the matrix-particle interface produce an initial stage of pronounced work hardening, while cold-rolled samples show partial recovery at room temperature which reduces the yield strength. Both phenomena lead to anomalous hardening. Ascast thin slabs of $\mathrm{Al}-13 \% \mathrm{Sn}-2.5 \% \mathrm{Si}-1 \% \mathrm{Cu}$ were cold rolled and annealed at $300^{\circ} \mathrm{C}$ for $60 \mathrm{~min}$. Tensile tests were performed along the rolling direction and micro-indentation tests executed along the normal, transverse and rolling direction. Tensile curves were fitted by several conventional hardening relations, of which the Voce-relationship provided the best flexibility in dealing with the non-standard hardening behaviour. Use of the fitted data in a finite-element model for microindentation provided a reasonable approximation to the measured load-displacement curves but material anisotropy is an overriding factor which was not accounted for. Evidence of creep was clearly present both in the loading stage as in the constant load part of the indentation curves.

Keywords: indentation, reverse modelling, finite elements, creep, anisotropy.
\end{abstract}

\section{Introduction}

Al-Sn alloys are used as the tribological component of journal bearings for light combustion engines. Although historically a fairly broad amount of compositions has been normalised [1], nowadays two alloys dominate the market, being SAE 783 (Al-19\%Sn-1\%Cu by weight) and SAE 788 (Al-12\%Sn-2.5\%Si-1\%Cu), the 
latter showing higher resistance than the former due to the presence of Silicon and AlFeSi-intermetallic particles and a lower Sn-content.

Wear in ductile tribo-alloys is dominated by severe plastic deformation [2-8] and ductility is one of the main properties to optimise in these products. However, strength determines the load bearing capacity of the alloy and stronger alloys allow for higher specific cylinder pressures in the engine and reduced dimensions, which reduce the mechanical losses caused by viscous dissipation in the lubricant. The precise knowledge of the constitutive behaviour of the alloys also is essential for the modelling of wear tests.

For research purposes and product optimisation, one can usually rely on tensile tests to determine the essential data required to describe the constitutive behaviour of these alloys. However, in industry, the Al-Sn alloys are bonded to a steel backing sheet by means of a high rolling reduction during the first processing steps. To compare the properties of the industrially produced bimetal strip to laboratory produced free-standing alloy strip, the measurement of load vs. displacement curves during indentation is a useful tool. Also the characterisation of the tribologically modified surface layer [2-8], with thickness between 1 and $10 \mu \mathrm{m}$, needs to rely on this technique.

Relationships between indentation hardness and tensile properties have been extensively studied by Tabor since the 1950s [9]. The extended use of instrumented indentation tests (see e.g. Oliver and Pharr [10]) has significantly increased the amount of information that can be obtained in such a test. Dimensional analysis reveals that the functional form of the indentation curve is limited to well-defined physical relationships and that the precise influence of constitutive equations and the geometry of the test are convoluted into the parameters that describe the curve [11]. For a self-similar indenter (pyramid or cone) and an elastoplastic material, the load $(P)$ vs. displacement $(h)$ follows:

$$
P=C_{l} h^{2}
$$

The (elastic) unloading curve is often described as:

$$
P=C_{u}\left(h-h_{r}\right)^{\beta}
$$

with $h_{r}$ the residual indentation depth. While (1) can be proven mathematically and $C_{l}$ is a material constant, (2) is only a fitting equation [11] and $C_{u}$ and $\beta$ are not material constants. These equations do not take into account pile-up or sinkin of the material around the indenter. In this case, the contact area is given by:

$$
A=c h^{2}
$$

where $c$ depends on the indenter geometry alone. For a power-law hardening elastoplastic solid $[11,12]$, the yield strength can be described as:

$$
\sigma=\sigma_{r e f}\left(1+\frac{E}{\sigma_{r e f}} \varepsilon\right)^{n} \quad \varepsilon \geq \frac{\sigma_{r e f}}{E}
$$

Depending on the amount of hardening, the material may pile-up or sink-in around the indenter and the true contact area is distinct from what is predicted from the indenter geometry alone. To do so, one can execute a large number of finite element simulations in which the material parameters are systematically varied, thereby constructing a database of indentation curves to which the 
measured curve can be fitted [13, 14]. Alternatively, one describes the relationship between material parameters and geometric variables by means of a set of non-dimensional functions $[12,15,16]$. To do so, the empirical equations are fitted to a large number of finite-element simulations. This allows reducing the inverse problem to the solution of a set of nonlinear equations with restrictions. Both approaches present different strategies for the numerical solution of the same problem.

For strain-rate sensitive materials, (4) is insufficient to describe the material behaviour. In the case of power-law creep $[17,18]$, the strain rate is given by:

$$
\frac{\dot{\varepsilon}}{\dot{\varepsilon}_{0}}=\left(\frac{\sigma}{\sigma_{0}}\right)^{m}
$$

or

$$
\frac{\dot{\varepsilon}}{\dot{\varepsilon}_{0}}=\left(\frac{\sigma-\sigma_{t}}{\sigma_{0}}\right)^{m}
$$

where $\dot{\varepsilon}_{0}$ and $\sigma_{0}$ are a reference strain rate and reference stress, $m$ is the strain rate sensitivity exponent and $\sigma_{t}$ a threshold stress below which creep does not occur. Either for (5) or for (6), dimensional analysis shows that the loaddisplacement curve can be described by:

$$
P=C_{c r} h^{\frac{2}{m+1}}
$$

where $C_{c r}$ depends on the indenter geometry, the loading rate and material parameters but not on $h$ or $P$. One can also obtain the expression for the displacement under constant load [11]:

$$
h(t)=\left(\frac{2}{m}\right)^{\frac{m}{2}}\left(\frac{\sigma_{0}}{\dot{\varepsilon}_{0}^{m}} \frac{C_{c l}}{P}\right)^{-\frac{1}{2}} t^{\frac{m}{2}}
$$

where $C_{c l}$ is a constant which depends on $\mathrm{m}$ and the indenter geometry but not on the variables $h$ and $t$ and $P$ is the constant load applied.

\section{Models}

To the authors' experience, standard software provided with testing equipment may be unreliable if nonstandard materials have to be analysed. Therefore, force-displacement data were loaded in the commercial Mathematica ${ }^{\circledR}$ software and determination of tensile data as well as the fitting of constitutive relations performed by means of the numerical routines available in the package. Yield stress was defined as the $\sigma_{0.2}$ limit. Fitting of the true stress - true strain curves was performed in the interval of strains corresponding to $\sigma_{0.2}$ and the ultimate tensile stress $\sigma_{\mathrm{u}}$. Apart from the modified Ludwik equation (4), the time and temperature independent part of the Johnsonn-Cook equation (9) and the Voce equation (10) were tested:

$$
\sigma=k\left(1-b \varepsilon^{n}\right)
$$




$$
\sigma=k\left(1-b e^{-n \varepsilon}\right)
$$

(9) has the advantage that it is directly available in the ABAQUS ${ }^{\circledR}$ finite element software although (4) is sometimes considered to provide better fits. The main advantage of (10) is that the stress saturates at high values of $\varepsilon$, while (4) and (9) may severely overestimate the stress at the high strain values observed directly underneath the indenter. In the present work, (9) was used for the finite-element simulations, but future work in this area will use (10). The modelling parameters are taken as the average of the parameters obtained from 3 tensile tests.

Forward modelling of the indentation tests was performed by means of the finite-element model shown in Fig. 1, using ABAQUS Explicit ${ }^{\mathbb{B}}$, where onequarter of a Vickers indentation test is shown. Material density was increased by a factor of $10^{6}$ to reduce the number of time increments in the explicit simulation [19]. This did not introduce any noticeable dynamic effects. Hourglassing due to reduced integration and mass lumping [19] was carefully checked and found to be of no importance in the simulations. Although the mesh used here is coarse compared to what is often presented in literature [12, 18], no modification of the results was obtained upon further refinement.

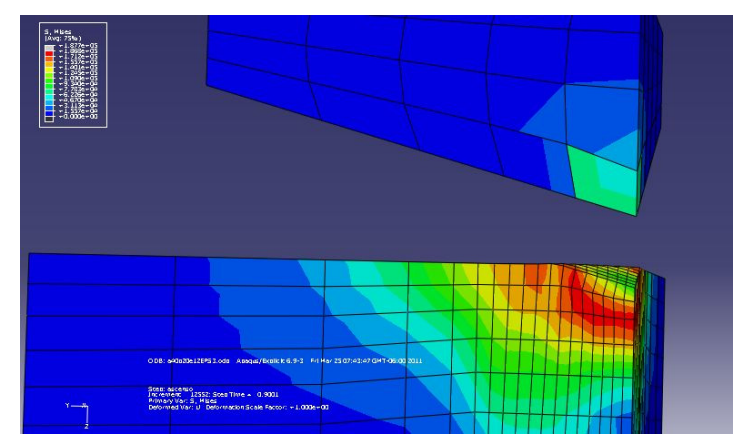

Figure 1: Mesh and residual stress distribution for an indentation test on a sample rolled to an equivalent strain of 3 and annealed for $60 \mathrm{~min}$ at $300^{\circ} \mathrm{C}$.

Reverse analysis was performed following the work of Dao et al. [12]. For details, the reader is referred to the original publication, where the nondimensional functions $\Pi_{1}$ to $\Pi_{5}$ are listed in the appendix. To determine the constitutive parameters in eq. (4), one starts by defining the relationship between the maximum indentation depth $h_{m}$ and the residual depth $h_{\mathrm{r}}$ by the use of a nondimensional function:

$$
\frac{W_{p}}{W_{t}}=\Pi_{5}\left(\frac{h_{r}}{h_{m}}\right)
$$

It is considered that the relationship of plastic work $W_{p}$ over total work $W_{t}$ can be measured more precisely than $h_{r} / h_{m}$. One then solves for the maximum contact area $A_{m}$ (taking into account pile-ups and sink-ins) by setting: 


$$
A_{m}=\left(P_{m} c^{*}\right)^{2}\left(\left.\frac{d P}{d h}\right|_{h=h_{m}} \Pi_{4}\left(\frac{h_{r}}{h_{m}}\right)\right)^{-2}
$$

with $P_{m}$ equal to the maximum load, $c^{*}$ a constant which is equal to 1.2105 for the Vickers indenter and the derivative represents the slope of the unloading curve evaluated at $h_{m}$. Then the reduced Young's modulus $E^{*}$ is found by

$$
E^{*}=\left.\frac{1}{c^{*} \sqrt{A_{m}}} \frac{d P}{d h}\right|_{h=h_{m}}
$$

with:

$$
E^{*}=\left(\frac{1-v}{E}+\frac{1-v_{\text {ind }}}{E_{\text {ind }}}\right)
$$

where $E\left(E_{\text {ind }}\right)$ and $v\left(v_{\text {ind }}\right)$ represent the Young's modulus and Poisson's coefficient of the material (indenter). $E_{\text {ind }}$ and $v_{\text {ind }}$ were taken equal to $1100 \mathrm{GPa}$ and 0.07 as in [12], $v$ was set equal to 0.35 , the conventional value for Al. The value of the reference stress $\sigma_{0.033}$ (the stress at $3.3 \%$ true strain in uniaxial tension) and of the strain hardening exponent $n$ (eq. (4)) can then be found by numerically solving (15) and (16)

$$
\begin{gathered}
C_{l}=\sigma_{0.033} \Pi_{1}\left(\frac{E^{*}}{\sigma_{0.033}}\right) \\
\left.\frac{1}{h_{m} E^{*}} \frac{d P}{d h}\right|_{h=h_{m}}=\Pi_{2}\left(\frac{E^{*}}{\sigma_{0.033}}, n\right)
\end{gathered}
$$

$E, \sigma_{0.033}$ and $n$ provide sufficient data to determine (4).

\section{Experiments}

An industrially cast slab of $25 \mathrm{~mm}$ thickness with nominal composition of Al$12 \% \mathrm{Sn}-2.5 \% \mathrm{Si}-0.7 \% \mathrm{Cu}-1.5 \mathrm{~Pb}$ and impurities of iron and manganese was machined down to $15 \mathrm{~mm}$ and cold rolled in steps of $10 \%$ to an equivalent logarithmic strain of 3. Half of the samples were annealed for 60 minutes at $300^{\circ} \mathrm{C}$ with temperature control confirmed by thermocouples welded on reference samples. Samples of reduced dimensions were produced according to ASTM norm E8, with uniform gauge length of $30 \mathrm{~mm}$ and width of $6 \mathrm{~mm}$. Tests were executed on a Shimadzu AG-100KNX universal testing machine and results corrected for machine compliance. Instrumented hardness tests were executed on polished sections of as-rolled and annealed samples mounted in cold-curing resin (Buehler epo-quick ${ }^{\circledR}$ ). A typical microstructure of an annealed sample is given in Fig. 2. Instrumented indentation tests were executed on a Microphotonics Nanovea ${ }^{\circledR}$ platform with a Vickers pyramid. Loading and unloading rates were $8 \mathrm{~N} / \mathrm{min}$ up to $4 \mathrm{~N}$, the samples were held for $10 \mathrm{~s}$ at $4 \mathrm{~N}$. The equipment uses an optical height measuring technique, taking the undeformed sample surface as a reference, thereby eliminating effects of equipment compliance. Hardness measurements were executed perpendicular to normal, transversal and rolling direction (ND, TD, RD). 


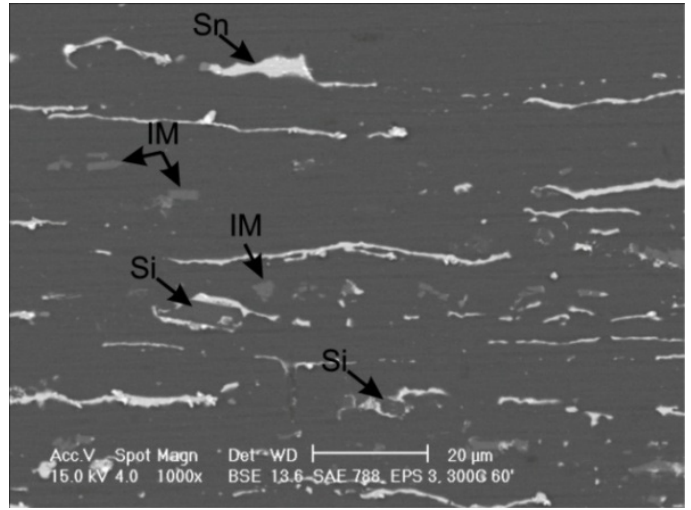

Figure 2: Backscattered electron image of an annealed sample in the RD/ND plane, showing elongated Sn-particles, intermetallic particles (IM) distributed in the Al-matrix and Si-particles.

\section{Results}

Two representative tensile curves (cold rolled/ cold rolled and annealed) are shown in Fig. 2a. As a reference, the straight line used to determine $\sigma_{0.2}$ is also shown. It is seen that the measured curves deviate from linear behaviour starting at very low stresses. One may reasonably argue that this is due to measuring errors associated to machine compliance or specimen clamping. This is not the case, because many other samples (steel, copper alloys) do not show this behaviour when measured on the same equipment under the same testing protocol. This behaviour is characteristic for the alloys studied here. Fig. 2 b. shows the corresponding true stress-true strain curves, together with a power law fit (4) and the Voce-equation fit (10). Although neither approximation is perfect, the Voce-equation is slightly better.
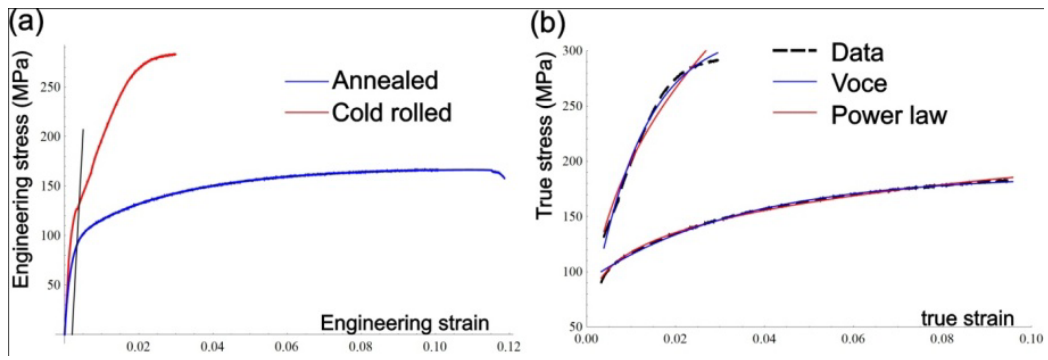

Figure 3: (a) shows the "raw" tensile curves for a cold-rolled and a coldrolled and annealed sample respectively. The thin black line corresponds to the determination of $\sigma_{0.2}$. (b) gives the true stress true strain curves together with the power-law fit (4) and the Voce fit (10). 
To compare the average indentation curves for $\mathrm{ND}$, TD and $\mathrm{RD}$ to the modelled curve from the finite element analysis, the loading curves were fitted to:

$$
P=C_{f i t}\left(h-h_{0}\right)^{\alpha}
$$

The difference between (17) and the theoretical curve (1) is justified for two reasons. Firstly, the material clearly shows creep; therefore, $\alpha$ will not be equal to 2.Secondly, the experimental procedure asks for the operator of the indentation equipment to decide where the loading curve supersedes the background noise. However, this point can never be exactly be determined, because as long as $\alpha>1$, both the value as well as the derivative of (17) become equal to 0 at $h_{0}$. Consequently, the "true" starting point of the loading curve will always be masked by the background noise around 0 . The "average" curve was obtained by shifting all curves by their $h_{0}$-values toward the origin and then averaging the values of $C_{f i t}$ and $\alpha$ for each set. Similarly, the constitutive parameters used for the finite element model were obtained by fitting (9) to each individual tensile curve and averaging $k, b$ and $n$; the average values were then plugged into the finite element model. The result is shown in Fig. 4.

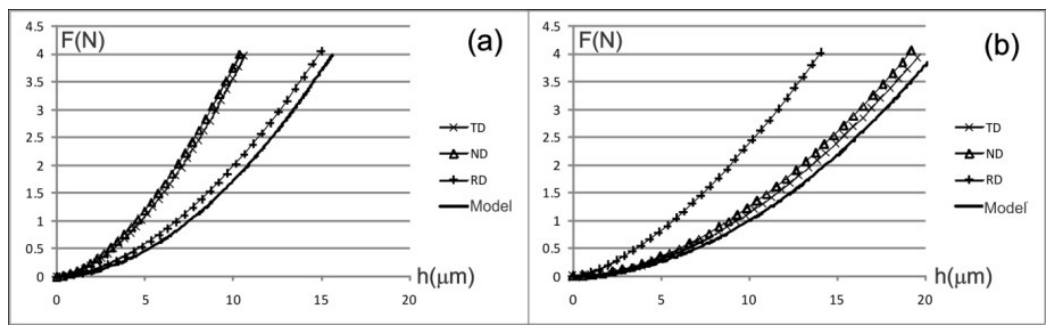

Figure 4: Average and modelled indentation curves for the as-rolled material (a) and the annealed material (b).

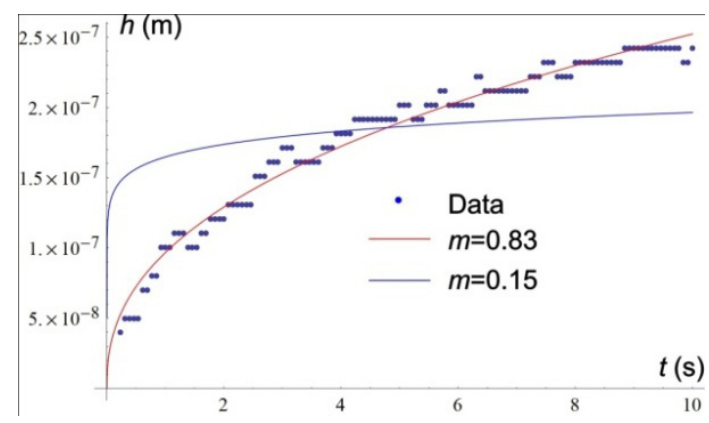

Figure 5: Creep data for an annealed sample. The red line is found by fitting (8) with $m$ a free parameter, the blue line takes $m$ as found from the fitted exponent in (17), using (7). 
Reverse modelling of the tensile properties by means of the non-dimensional analyses presented above were generally unsuccessful. They are worth mentioning only because they allow us to identify the main reasons for discrepancy between the reverse analysis and experiments observed in the present work and the generally good results mentioned in classical works on this topic $[12,15,16]$. Values of the strain hardening exponent $n$ (eq. (4)) varied between 0.15 and 0.6 , with no sensible average to be determined for both processing conditions. The strain rate sensitivity exponent $m$, as determined from (17), varied between negative values (which have no physical meaning) to 0.6 . An approximate value of $0.2 \pm 0.1$ can be proposed for both conditions after exclusion of the most extreme values. The value of $E$ was typically three to four times lower than the value for $\mathrm{Al}(70 \mathrm{GPa})$.

According to (8), the constant loading part of the curve can, theoretically, provide some additional information on $m$. The degree to which this is correct is shown in fig. 5. The value of $\alpha$ estimated from the loading curve is 1.7, which leads to $m \approx 0.15$. Least squares fits to (8) are shown, one using the value of $m$ obtained from the fitted loading curve (17) and one from the measured curve, with $m$ considered a parameter. In the latter case, $m$ was found to be approximately equal to 0.83 .

\section{Discussion}

By definition, a model is based on assumptions. Testing a model thus implies two aspects; the first one is whether the model is self-consistent, i.e. if data which are consistent with the assumptions are also consistent with the results of the model and vice versa. This question also addresses uniqueness. The second aspect is whether the phenomenon to be modelled conforms to the model, which is a test on whether the assumptions describe the phenomenon to sufficient accuracy. To verify this aspect, one has to perform experiments, introducing a third consideration, related to the relevance and accuracy of the measurements. It has already been demonstrated [11] that the first aspect is not completely addressed by the reverse model. This is probably not the most severe problem if data on material behaviour are available from independent tests. The following is a point-by-point review of the differences between experiment, model and theory which affect the interpretation of the results:

a) The reverse analysis is critically dependent on the validity of (1). If $\alpha=2$ in (17), then there is little liberty for $h_{0}$ and $C_{f i t}$ in the least-squares fit. Also, if $h_{0}$ can be determined independently, then the other two parameters are well defined. However, the onset of contact loading is drowned in the noise of the measurements. As a matter of fact, for each of 87 curves, the operator defined the onset of loading at a point where the slope of the curve was already positive.

If one leaves all three parameters free in eq. (17), then it is found that variations of $\alpha$ from 1.7 to 2 implicate very reasonable variations of $h_{0}$, but changes in $C_{f i t}$ by a factor of 10 . In the unloading curve, the effects are even more drastic. The value of $\beta$ may vary from 1.2 to 4 by the choice of point (c) 
as shown in Fig. 6, which results in a variation of $C_{u}$ by three orders of magnitude. The value of the slope at $h=h_{m}$ is almost not affected by these variations. $h_{r}$ is significantly more sensible and therefore, it is better to use (11) to determine $h_{m} / h_{r}$.

The true problem in finding the tensile parameters by means of reverse analysis of indentation data is therefore the difference between $C_{f i t}$ and $C_{l}$. This is demonstrated in Fig. 6. 6b. shows how the value of $n$ can take one, two, three or no solution for different values of $E^{*}$ and the slope at $h_{m}$. As mentioned before, the latter value is fairly insensible on the details of the fitting procedure, but $\sigma_{0.033}$ is strongly affected by $C_{l}$ (eq. (15)).

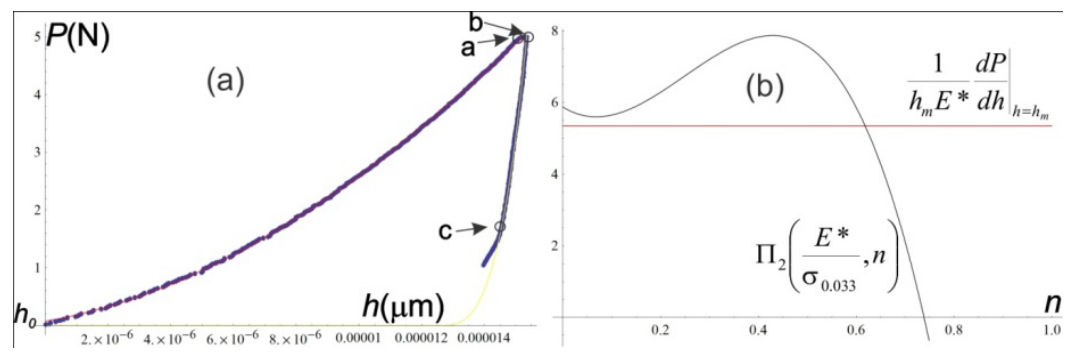

Figure 6: (a) shows the fit for the loading curve (red) and unloading curve (yellow). Point a indicates the end of loading, $\mathbf{b}$ is the start of unloading, the interval $[\mathrm{a}, \mathrm{b}]$ is the constant loading part. Point $\mathbf{c}$ is the final fitting point for the unloading curve (2). Curve fitting is almost independent on $\mathbf{a}$ and $\mathbf{b}$ but varies strongly with $h_{0}$ and $\mathbf{c}$. (b) shows the behaviour of equation (16).

b) The reverse model was constructed under the assumption that (4) holds. The tensile curves show significant deviations of this assumption. None of the classical fits used for tensile data is accurate for the present material. Both the as-rolled and the annealed material have a low yield strength which corresponds to significant non-linear behaviour before the $0.2 \%$ proof stress is reached. This behaviour is actually quite common in particle-reinforced materials.

As was shown by Brown and colleagues [20,21], the zones close to the particles will reach their yield strength before the overall yield strength of the material is reached, leading to stronger local hardening causing a significant increase of stress in this stage. Once the plastic zones expand over the entire sample, "normal" hardening takes over. In the present material, it is seen that intermetallic particles are generally clustered. Such clusters have a different effect on hardening than homogeneously distributed particles [22]. Due to the size of the clusters as compared to the size of the plastic zone during indentation, this may induce a length scale into the problem, which in turn would invalidate the dimensional analysis which leads to (1).

The presence of hard precipitates has also been shown to produce an upward convex slope of the strain hardening curve $[23,24]$, as is seen in the 
cold-rolled materials. It is known that prestraining and the presence of large atoms such as Sn [24-26], enhance the precipitation kinetics in a significant way. Al-Cu alloys with $\mathrm{Sn}$ additions show pronounced room-temperature ageing. In the present material, the amount of $\mathrm{Cu}$ is too small to cause significant age-hardening and its role is generally considered to be a solid solution hardener. The present authors have also considered the effect of room-temperature recovery. While such a process would reduce the overall dislocation density, the presence of solutes $(\mathrm{Cu}, \mathrm{Fe}, \mathrm{Mg}, \mathrm{Sn})$ would prevent polygonisation and subgrain growth. The strong initial strain-hardening stage in the cold-rolled stage can then be attributed by the accumulation of new dislocations on the old subgrain boundaries until reaching the original asrolled dislocation density, from where the material shows a hardening rate which corresponds to what can be expected for the corresponding rolling strain.

c) If diffusional processes such as precipitation and recovery are present at room temperature, then it is no surprise that the material also shows roomtemperature creep. The practical problems encountered in determining $m$ from the loading curve would be easy to solve if an independent determination of $m$ could be made. As seen in Fig. 5, the value of $m$ estimated during the constant loading part of the indentation is a clear overestimation. This is explained by the fact that the creep observed here is still in a transient stage. Such transients are generally neglected in any treatment of metal viscoplasticity, but were clearly confirmed in experiments by Sinha [27].

d) Limited studies on the effect of anisotropy on indentation behaviour are available [28]. These refer principally to morphological anisotropy, i.e. anisotropy based on an elongated grain shape or aligned short fibres in a metal-matrix composite. While the present alloy shows morphological anisotropy in the form of Sn-ribbons aligned with the rolling direction, the volume fraction of $\mathrm{Sn}$ is too small to cause significant anisotropy. Moreover, the effect of anisotropy is different in the as-rolled material as compared to the annealed material, as RD is the softest direction in the former and the hardest one in the latter. This can be explained only by the change in the crystallographic texture during annealing, which means that, in order to model such behaviour, simple anisotropic yield criteria (e.g. the Hill criterion [29]) will be insufficient and crystal plasticity based models [30] must be explored. For the cold rolled material, changes of strain path between rolling deformation and the spherical expansion mode associated to indentation will also have a significant effect [31].

e) As to the relative importance of the topics mentioned here, it appears to the authors that the crystallographic anisotropy of the material is one of the main factors to be addressed. The fact that the difference between some of the modelled and measured curves is smaller than the difference between the measured curves themselves is a clear indication of the impact of this effect. Creep seems to be a problem that can be countered more easily. First, it is not so difficult to consider power law creep in the finite element models. Second, in spite of the transient behaviour observed in the constant loading part of the 
present indentation tests, there is no reason why the duration of this stage cannot be extended, such as to separate transition from steady-state. Once $m$ is measured with better precision, the determination of the other parameters in the loading curve (17) becomes significantly easier.

\section{Conclusions}

Forward and reverse modelling of the constitutive parameters for an Al-Sn-Si metal-metal composite was performed based on instrumented indentation and tensile tests. Classical equations to describe the tensile behaviour of metals were found to be imprecise for the actual materials, but the finite element model used to predict the indentation curve gave reasonable results. Using isotropic plasticity theory, it was not capable of reproducing the large differences in indentation curves caused by the anisotropy of the material. The reverse analysis was not applicable to the present material, due to the nonstandard tensile behaviour and more importantly, due to the presence of creep which invalidates the fitting procedures inherent to the method. The effect of creep seems to be a problem that can be solved by small modifications of the experimental method and fitting procedures, while the effect of anisotropy and strain path changes will require serious additional modelling efforts. The observation that standard methods cannot be applied to nonstandard materials is nothing more than an expression of common sense. To be or not to be standard is the question. Most likely, many precipitation-hardening aluminium alloys are not.

\section{Acknowledgements}

The authors would like to thank A. Pérez and E. Nava for their involvement in the practical elaboration of this work. Financial support by DGAPA under grant PAPIIT IN120209 and by CONACyT under grants CONACYT-SEP 061259 and CONACYT-SEP 083723 is greatly acknowledged.

\section{References}

[1] Kingsbury, G.R. Friction and Wear of Sliding Bearing Materials. Metals Handbook, 10 ${ }^{\text {th }}$ Edition, Vol. 18. ASM International 1992.

[2] Meshi, L., Samuha, S., Cohen, S.R., Laikhtman, A., Moshkovich, et al. Dislocation structure and hardness of surface layers under friction of copper in different lubricant conditions. Acta Mater., 59(1), pp. 342-348, 2011.

[3] Kolubaev, A., Tarasov, S., Sizova, O., Kolubaev E., Scale-dependent subsuface deformation of metallic materials in sliding, Tribology International 43 pp. 695-699, 2010.

[4] Zhang, Y.S., Han, Z., Lu, K. Fretting wear behavior of nanocrystalline surface layer of copper under dry condition, Wear 265 pp. 396-401, 2008.

[5] Schouwenaars, R., Jacobo, V.H., Ortiz, A. Microstructural aspects of wear in soft tribological alloys. Wear, 263(1-6), pp. 727-735, 2007. 
[6] Ueda, M., Uchino, K., Senumura, T. Effects of carbon content on wear property in pearlitic steel. Mater. Sci. Forum, 426-432 pp, 1175-1180, 2003.

[7] Kapoor, A., Franklin, F.J. Tribological layers and the wear of ductile materials. Wear, 245 pp. 204-215, 2000.

[8] Rigney, D.A., Naylor, M.G.S., Divakar, R., Ives, L.K. Dislocation structures by sliding and by particle impact, Mater. Sci. Eng. 81 pp. 409425, 1986.

[9] Tabor, D. The hardness of metals, Clarendon Press, 1951.

[10] Oliver W.C., Pharr G.M. An improved technique for determining hardness and elastic modulus using load and displacement sensing indentation experiments. J. Mater. Res. 7 pp.1564-1583, 1992.

[11] Cheng, Y.T, Cheng, C.M. Scaling, dimensional analysis and indentation measurements. Mater. Sci. Eng. R. 44 pp. 91-149, 2004.

[12] Dao, M., Chollacoop, N., van Vliet, K.J., Venkatesh, T.A., Suresh, S., Computational modeling of the forward and reverse problems in instrumented sharp indentation. Acta mater. 49 pp. 3899-3918, 2001.

[13] Tardieu N., Constantinescu, A. On the determination of elastic coefficients from indentation experiments. Inverse Problems 16 pp. 577-589, 2000.

[14] Constantinescu, A., Tardieu, N. On the identification of elastoviscoplastic constitutive laws from indentation tests. Inverse Problems in Science and Engineering 9, pp. 19 - 44, 2001.

[15] Giannakopoulos A.E., Suresh S. Determination of elastoplastic properties by instrumented sharp indentation. Scripta Mater. 40(10), pp. 1191-1198, 1999.

[16] Bucaille, J.L., Stauss S., Felder, E. Michler, J. Determination of plastic properties by instrumented indentation using different sharp indenters. Acta mater. 51, pp. 1663-1678, 2003.

[17] Ma, X., Yoshida, F., Shinbata, K. On the loading curve in microindentation of viscoplastic solder alloy Mater. Sci. Eng. A344 pp. 296-299, 2003.

[18] Kermouche, G., Loubet, J.L., Bergheau, J.M. Cone indentation of timedependent materials: The effects of the indentation strain rate. Mechanics of Materials 39 pp. 24-38, 2007.

[19] Zienkiewicz, O. C., Taylor R. L., The Finite Element Method. ButterworthHeinemann 2006.

[20] Brown, L.M., Stobbs, W. Work-hardening of cu-silica. pt. 1. model based on internal stresses with no plastic relaxation. Philos. Mag. A. 23, pp. 1185-1199, 1971.

[21] Brown, L.M., Clarke, D.R. Work hardening due to internal stresses in composite materials. Acta metal. 23, pp. 821-830, 1976.

[22] Wilkinson, D.S., Pompe, W., Oeschner, M. Modelling the mechanical behaviour of heterogeneous multi-phase materials. Prog. Mater. Sci. 46, pp. 379-405, 2001.

[23] Kocks, U.F. Mecking, H. Physics and phenomenology of strain hardening: the FCC case. Prog mater. sci. 48 pp. 171-273, 2003. 
[24] Fribourg, G., Bréchet, Y., Deschamps A., Simar A. Microstructure-based modelling of isotropic and kinematic strain hardening in a precipitationhardened aluminium alloy Acta Mater. 59, pp 3621-3635, 2011

[25] Silcock, J.M. and Flower, H.M. Comments on a comparison of early and recent work on the effect of trace additions of $\mathrm{Cd}$, In or Sn on nucleation and growth of $\theta^{\prime}$ in Al-Cu-alloys. Scripta mater. 46 pp. 389-394, 2002.

[26] da Costa Teixeira, J., Bourgeois, L., Sinclair C.W., Hutchinson C.R. The effect of shear-resistant, plate-shaped precipitates on the work hardening of $\mathrm{Al}$ alloys: Towards a prediction of the strength-elongation correlation. Acta Mater. 57, pp. 6075-6089, 2009.

[27] Sinha N.K. Viscous and delayed-elastic deformation during primary creepusing strain relaxation and recovery test. Scripta Mater. 48 pp. 1507-1512, 2003.

[28] Yonezu, A., Yoneda, K. Hirakata, H., Sakihara, M., Minoshima, K. A simple method to evaluate anisotropic plastic properties based on dimensionless function of single spherical indentation-application to $\mathrm{SiC}$ whisker-reinforced aluminum alloy. Mater. Sci. Eng. A. 527, pp. 76467657, 2010.

[29] Hill, R. The Mathematical Theory of Plasticity, Oxford University Press, Oxford, 1950.

[30] Van Houtte, P., Li, S., Seefeldt. M, Delannay, L. Deformation texture prediction: from the Taylor model to the advanced Lamel model. Int. J. Plasticity 21, pp. 589-624 2005.

[31] Li, S., Hoferlin, E, Van Bael, A., Van Houtte, P., Teodosiu, C. Finite element modeling of plastic anisotropy induced by texture and strain-path change. Int. J. Plasticity 19 pp. 647-674, 2003. 